\title{
Resistance force pulsations of annular flow in the Couette-Taylor system with counter-rotating cylinders
}

\author{
Nikolay Miskiv ${ }^{1,2, *}$, Anatoly Serov ${ }^{1,3}$, Aleksandr Nazarov $^{1,2}$ and Valery Mamonov ${ }^{1}$ \\ ${ }^{1}$ Kutateladze Institute of Thermophysics, 630090 Novosibirsk, 1 Acad. Lavrentiev ave., Russia \\ ${ }^{2}$ Novosibirsk State University, 630090 Novosibirsk, 1 Pirogova str., Russia \\ ${ }^{3}$ Novosibirsk State Technical University, 630073 Novosibirsk, Prospekt K. Marksa, Russia
}

\begin{abstract}
The paper presents the results of an experimental study of the Couette-Taylor flow fluctuations in a ring channel with oppositely rotating multicylinder rotors. Experiments were carried out using water-glycerine solutions as a working fluid. The rotation resistance moment and its pulsations were investigated, using the system for measuring the torque resistance of rotation of rotors, made in the form of a digital dynamometer based on a tension sensor. The investigations made it possible to establish that the classic dependence of the appearance of Taylor vortices is observed in the slit flow of a multicylinder system rotating oppositely. It was shown that in the range of Reynolds numbers $\operatorname{Re}=(100-500)$, pulsations of dissipative processes with variable frequency and amplitude up to $10 \%$ of the mean value of rotation resistance are observed.
\end{abstract}

\section{Introduction}

At present a great practical interest are investigations related to the state of the flow in the slit space, when the coaxial cylinders rotate towards each other with given angular velocities. The results of such studies can be very useful for constructing an effective wind heat generator and a separator for oil chemistry.

Mallock (1888, 1896) and Couette (1890) have published a large number of experimental and theoretical studies of the flow between concentric rotating cylinders (the Couette circular flow) since the first studies. In particular, in [1] the system formed by two coaxial independently rotating cylinders was investigated. Based on the conducted studies, the authors presented a detailed map of flow regimes, in the annular ring between cylinders rotating relative to each other, as functions of the Reynolds numbers of the inner and outer cylinders. The optical method used by the authors made it possible to describe the behavior of pulsating structural formations that arise in the annular ring in a wide range of Reynolds number changes.

The physics of dissipative processes between fluid layers or between the surface of a solid and the surface of a liquid in the slit space of rotating cylindrical bodies has not been

\footnotetext{
* Corresponding author: nikerx@gmail.com
} 
sufficiently studied. There are various theories describing these processes, however, experimental data for creating a single model is not enough. The developed experimental setup, the apparatus and the measurement technique made it possible to perform studies of the dependence of the rotation resistance torque on the rotational speed and viscosity of the working fluid. The obtained experimental data make it possible to estimate the efficiency of heat generation due to dissipative processes in the liquid flow in a multi-gap space and to determine the algorithm for calculating the structure, working fluid and operating modes of the heat generator.

\section{Experimental setup}

Fig. 1 shows the scheme of the experimental setup. The setup allows one to investigate the influence of the working fluid parameters, the angular velocity of rotation, and the associated structure of the fluid flow in the gaps of the multicylinder rotor system, by the value of resistance to counter rotation.

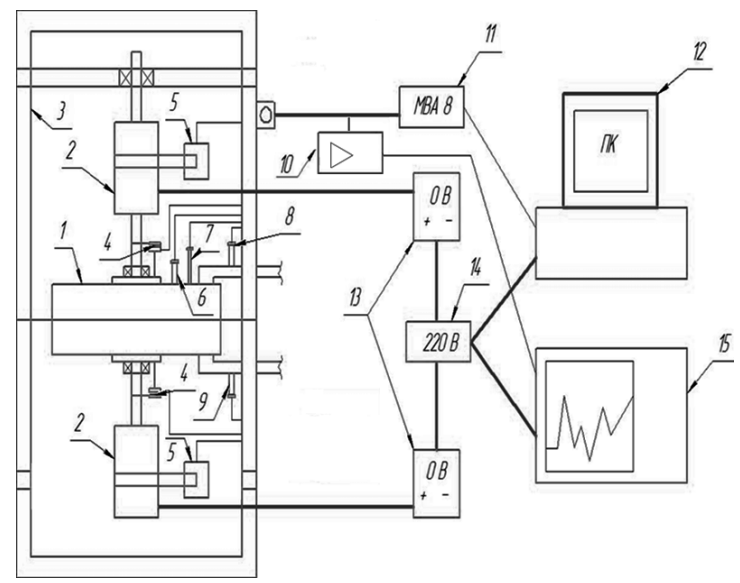

Fig. 1. The scheme of the experimental setup (1 - heat-insulated body, 2 - electric motor, 3 - frame, 4 - RPM sensor, 5 - tension sensors, 6 - body temperature sensor, 7 - liquid temperature sensor, 8 inlet temperature sensor, 9 - outlet temperature sensor, 10 - tension sensor amplifier, 11 - signal converter, 12 - computer, 13 - laboratory autotransformers, 14 - power supply, 15 - oscilloscope).

Spinning of the rotors is carried out by two electric motors with a variable speed. Opposite rotor block constructively consists of two identical rotors, inserted in the annular gaps of each other and forming a system of cylindrical annular channels. The name of the rotors is conditional, since both rotate relative to the block body. All block elements are made of aluminum alloy. The "rotors" rotating against each other form a coaxial multicylinder system consisting of 13 annular cylindrical channels with a width of $\sim 2.75 \mathrm{~mm}$ and an annular gap height of $50 \mathrm{~mm}$, and a ring diameter of 203 to $315 \mathrm{~mm}$. The system is filled with a working viscous liquid with predetermined properties. The working fluid is a waterglycerin mixture, the parameters of which change, heating when the rotors rotate due to large shear stresses in the annular channels. Table 1 shows the parameters of the mixture with a water concentration of $17 \%$.

Table 1. The parameters of working fluid.

\begin{tabular}{|c|c|c|c|c|}
\hline № & $\begin{array}{c}\text { Water } \\
\text { concentration, } \\
\%\end{array}$ & $\begin{array}{c}\text { Temperature } \\
\mathrm{T},{ }^{0} \mathrm{C}\end{array}$ & $\begin{array}{c}\text { Kinematic } \\
\text { viscosity } \\
v, \mathrm{~m}^{2} / \mathrm{s}\end{array}$ & $\begin{array}{c}\text { Density } \rho, \\
\mathrm{kg} / \mathrm{m}^{3}\end{array}$ \\
\hline 1 & 17 & $20-50$ & $(54-15) \cdot 10^{-6}$ & $1200-1183$ \\
\hline
\end{tabular}


Furthermore, the kinetic energy of the motor drive is completely converted into thermal energy. Selection of heat energy from the heat generator is carried out by circulating the working fluid in a closed loop containing the heat exchanger. The thermal power allocated by heat generator is calculated on the basis of the measured total moment of resistance to rotation of the "rotors" according to the formula:

$$
N=M \cdot \Omega_{\Sigma}
$$

where $N[\mathrm{~W}]$ - mechanical power, $M[\mathrm{~N} \cdot \mathrm{m}]$ - measured total torque, $\Omega_{\Sigma}\left[\mathrm{s}^{-1}\right]-$ relative angular velocity of rotation.

The determination of the thermal power from the measured total moment was confirmed experimentally [2]. The total torque of resistance to rotation was transferred through a lever arm of $110 \mathrm{~mm}$ length to the strain gauge of an analog dynamometer 5 (Fig. 1). The data from the dynamometer was recorded by a digital oscilloscope PC-Lab2000SE in the modes of recording the moment of resistance. The signals from the temperature sensors were fed through the microprocessor-based data acquisition and pre-processing unit 11 to the computer for final processing and storage. A more complete description of the installation and the measurement procedure can be found in [2].

\section{Results and discussion}

Resistance pulsations were studied in the range of small Reynolds numbers $\operatorname{Re}=(100-$ 500) of the fluid flow. In these regimes, wave vortex flows arise [1], which determine the pulsations in the power of the thermal processes in the gaps. The nonstationarity of the liquid flow with viscosity $54 \mathrm{cSt}$ in the heat generator at the opposite rotation mode with a constant angular velocity $\Omega=22 \mathrm{~s}^{-1}$ demonstrates the graph of the moment of resistance M on time, shown in Fig. 2. The axis $t$ is show in the logarithmic scale. The flow regime corresponds to $\operatorname{Re}=140$.

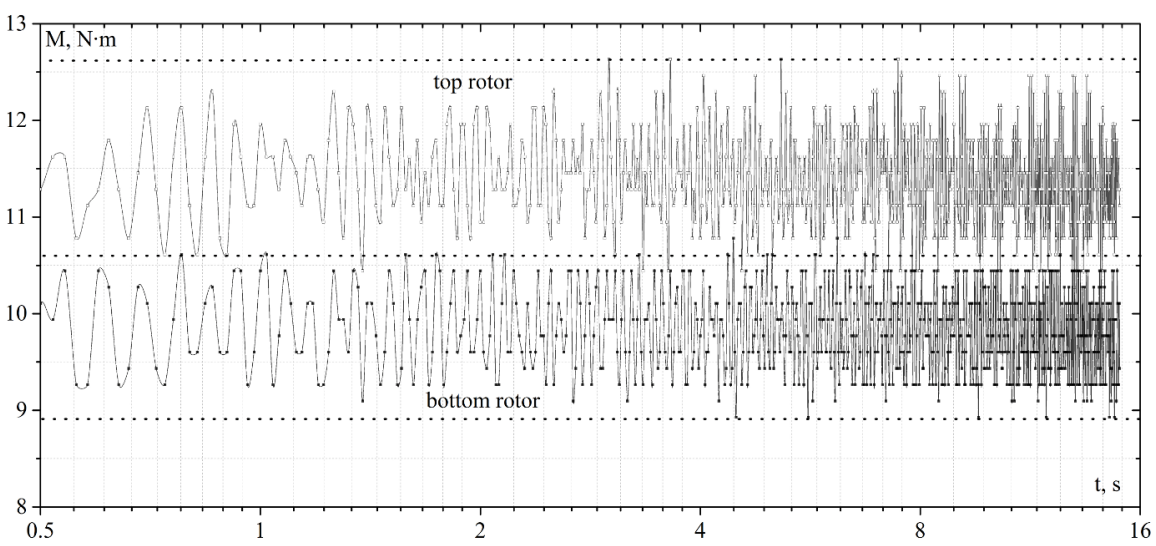

Fig. 2. A graph of torque pulsations for the upper and lower rotors in the opposite mode with a speed of $22 \mathrm{~s}^{-1}$ and a viscosity of $54 \mathrm{cSt}$.

It can be seen from the graphs in fig. 2 that the behavior of the resistance moment in rotors is stochastic, have a different frequency and amplitude varies by $\pm 10 \%$ of the mean value. An analysis of the data obtained shows that there are vortices in the upper and lower rotors in the liquid flow, and the vortex processes in the rotors are not connected with each other. 
Fig. 3a shows the dependence of the heat generator power on the Reynolds number for one constant angular velocity $\left(\Omega=22 \mathrm{~s}^{-1}\right)$ of rotation in single and opposite modes. The number Re was changed due to a change in viscosity in the range $v=(54-15) \cdot 10^{-6} \mathrm{~m}^{2} / \mathrm{s}$ when the water glycerin mixture was heated as a result of dissipative processes.
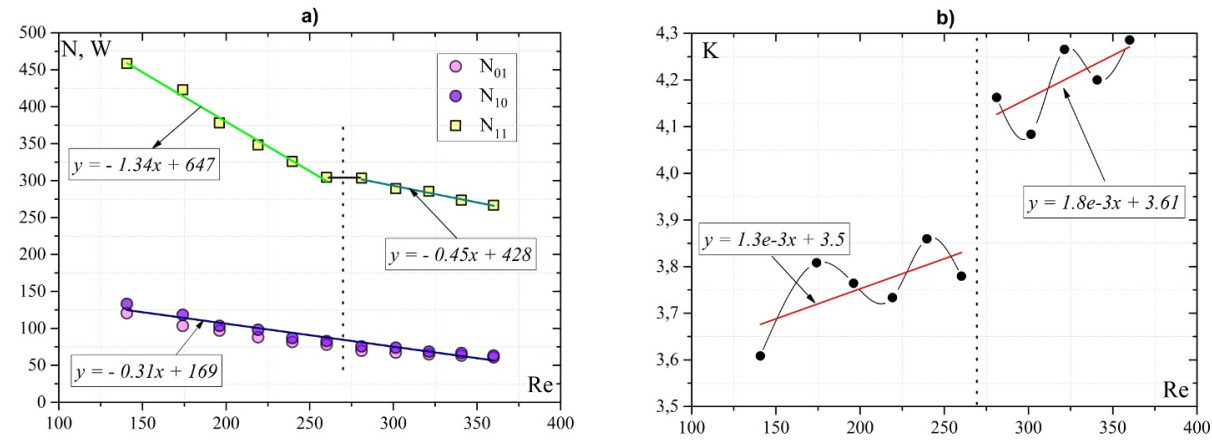

Fig. 3. Dependencies of the generator power (a) and the power ratio $\mathrm{K}$ (b) on the number Re. $\mathrm{N}_{11^{-}}$ counter rotation mode of the rotors, $\mathrm{N}_{01}$ and $\mathrm{N}_{10}$ - single rotation of the upper or lower rotors.

The data show that the heat output for the opposite rotation of $\mathrm{N}_{11}$ is more than three times higher than for single-rotation regimes $\mathrm{N}_{01}, \mathrm{~N}_{10}$ (fig. 3a). When analyzing the dynamics of the power behavior in the $\mathrm{N}_{11}$ mode, two regions can be distinguished: $\operatorname{Re}_{1}=$ (140-270) and $\operatorname{Re}_{2}=(270-350)$. The first region of Reynolds numbers corresponds to a laminar flow, the second is characterized by the presence of Taylor vortices in the flow of liquid [1]. The heat output for the first area is more than twice as high as for the second.

Figure $3 b$ shows the coefficient of the ratio of the heat output of the $\mathrm{N}_{11}$ mode to the average value of single-rotation regimes $\left(\mathrm{K}=\mathrm{N}_{11} / \mathrm{N}_{\mathrm{av0} 0}\right)$. The data show that for the $\mathrm{Re}_{2}$ region, the increase in thermal power with the opposite mode increases with respect to regimes $\mathrm{N}_{01}, \mathrm{~N}_{10}$, in comparison with the region $\mathrm{Re}_{1}$.

From the analysis of the experimental data, the following conclusions can be drawn:

- when measuring the resistance moment, practically at all Reynolds numbers in the ring flow, there are pulsations of the resistance force that corresponds to the vortex nature of the flow;

- the thermal power released under the opposite regime is more than 3 times higher than under single modes, which indicates that the opposite regime at one angular speed allows for more than triple heat output in smaller working volumes of the heat generator;

- the results of the research carried out can serve as a basis for future theoretical and experimental research in the development of efficient systems of alternative sources of thermal energy generation.

The reported research was funded by Russian Foundation for Basic Research and the government of the region of the Russian Federation, grant № 18-48-540009.

\section{References}

1. C.D. Andereck, S.S. Liu, H.L. Swinney, J. Fluid Mech., 164, 155-183 (1986)

2. V.N. Mamonov, A.D. Nazarov, A.F. Serov, T. and A., 23, 139-142 (2016)

3. A.F. Serov, V.N. Mamonov, V.I. Terekhov, A.D. Nazarov, Patent for invention, 2612237 (2017)

4. G. Schlichting, Boundary layer theory (Science, Moscow, 1969) 